\title{
Philonsorbonne
}

9 | 2015

Année 2014-2015

\section{L'objection « nationale » de David Miller aux théories cosmopolitiques sur la justice mondiale}

\section{Mathilde UNGER}

\section{(2) OpenEdition}

Journals

Édition électronique

URL : https://journals.openedition.org/philonsorbonne/736

DOI : 10.4000/philonsorbonne.736

ISSN : $2270-7336$

Éditeur

Publications de la Sorbonne

\section{Édition imprimée}

Date de publication : 1 janvier 2015

Pagination : 71-87

ISSN : 1255-183X

\section{Référence électronique}

Mathilde UNGER, «L'objection « nationale » de David Miller aux théories cosmopolitiques sur la justice mondiale », Philonsorbonne [En ligne], 9 | 2015, mis en ligne le 11 janvier 2015, consulté le 09 juin 2021. URL : http://journals.openedition.org/philonsorbonne/736 ; DOI : https://doi.org/10.4000/ philonsorbonne.736 


\title{
L'objection « nationale » de David Miller aux théories cosmopolitiques sur la justice mondiale
}

\author{
Mathilde UNGER
}

Dans le débat sur les théories de la justice distributive mondiale, les auteurs dits « cosmopolitiques ${ }^{1}$ » affirment que parce que les conditions de production des richesses sont devenues mondiales, que le capital comme le travail circulent de plus en plus librement à travers les frontières, il convient d'envisager la répartition des bénéfices produits et la protection des travailleurs à une échelle supranationale, voire mondiale. Ce texte s'intéresse à une objection formulée contre cette ambition « cosmopolitique » donnée à la justice sociale. Elle défend qu'en dépit de la mondialisation commerciale, de l'internationalisation du marché du travail et de l'ouverture des économies nationales aux investissements étrangers, le lieu de la justice sociale doit, lui, demeurer national.

David Miller a donné à cette idée qui relève de l'opinion commune une élaboration théorique ${ }^{2}$. Selon celle-ci, les principes de justice sociale sont nés et ont été rendus possibles à l'intérieur d'un contexte de solidarité national et doivent pour cette raison y rester. Commençons par préciser qu'il désigne par "justice sociale» la répartition des avantages et des désavantages créés par la coopération sociale entre les membres d'une

1. C. Beitz, Political and Theory International Relations, Princeton, N. J., Princeton University Press, 1979; T. Pogge, " An Egalitarian Law of Peoples », Philosophy and Public Affairs 23(3), summer 1994 ; A. Buchanan, «Rawls's Law of Peoples : Rules for a Vanishes Westphalian World », Ethics 110, n 4, 2000, p. 697-721; S. Caney, Justice beyond Borders : a Global Political Theory, Oxford, Oxford University Press, 2005.

2. D'un point de vue méthodologique, il défend d'ailleurs l'inclusion des croyances communes dans l'élaboration des principes de justice dans D. Miller, Principles of social justice, Cambridge, Mass., Harvard University Press, 1999. 
communauté (la répartition des richesses, des professions, l'accès à l'éducation ou à une couverture maladie, la distribution des honneurs et des prix, le droit aux loisirs, à un logement, mais aussi le service militaire, l'exercice de métiers dégradants, etc. ${ }^{3}$ ).

Même si les principaux textes de Miller sur la justice sociale sont antérieurs à ce débat sur les échelles de justice, ils éclairent les objections qu'il a plus récemment formulées contre «l'égalitarisme global ${ }^{4} »$. Il n’y aurait pas de sens, selon lui, à vouloir sortir les droits et les devoirs sociaux hors des frontières de l'État-nation puisque c'est le contexte national qui a dessiné leur contenu et qui doit donc continuer à délimiter leur domaine d'application adéquat. Miller accorde une attention particulière aux conditions matérielles et intellectuelles dans lesquelles la justice sociale a $\mathrm{pu}$ émerger comme concept et peut continuer à fonctionner comme un principe politique . $^{5}$.

Dans ses Principles of social justice ${ }^{6}$, Miller écrit ainsi :

«Ce n'est certainement pas un hasard si l'idée [de justice sociale] est apparue dans le contexte social et politique où elle est apparue: dans les sociétés libérales économiquement développées de la fin du XIX ${ }^{\mathrm{e}}$ et du début $\mathrm{du} \mathrm{XX}^{\mathrm{e}}$ siècle. Et de la même manière, les circonstances de son apparition pourraient suggérer les limites de son étendue. Si l'on essaie d'étendre le concept trop loin, on risque de découvrir que les conditions requises pour le faire fonctionner ne tiennent plus ${ }^{7} »$.

Ces « sociétés $^{8}$ » désignent pour lui des communautés politiques fermées. Parmi elles, les États-nations occupent une place particulière. En effet, lorsque les États sont aussi des «nations », c'est-à-dire des communautés soudées par une culture singulière et dont le lien avec un territoire persiste à travers l'histoire ${ }^{9}$, ils possèdent trois caractéristiques qui rendent à la fois possible et fructueuse l'application de principes de justice : les identités nationales créent des liens forts de solidarité, les cultures politiques nationales comprennent un ensemble de conceptions communes concernant la justice et, enfin, leur cadre institutionnel et leur pouvoir de sanction donnent à chacun de ses membres la garantie que les autres se plient à des sacrifices égaux ${ }^{10}$. L'État-nation constitue donc à la fois le contexte

3. Ibid., p. 7.

4. Pour cette critique, voir D. Miller, National Responsibility and Global Justice, Oxford/New York, Oxford University Press, 2007, chapitre 3.

5. D. Miller, op. cit., 1999, p. 2.

6. D. Miller, op. cit., 1999.

7. Ibid., p. 2.

8. Il entend par «société » l'équivalent de la structure de base pour John Rawls dans la Théorie de la justice.

9. D. Miller, On nationality, Oxford/New York, Clarendon Press/Oxford University Press, 1995, p. 27.

10. D. Miller, op. cit., 1999, p. 18-19. 
d'émergence et le lieu de justification des principes de justice sociale, ou plutôt, il en dessine le domaine d'application légitime car il en a été le berceau historique. Il n'est pas seulement le cadre historique dans lequel l'idée de justice sociale aurait germé, pour des raisons indépendantes, mais constitue l'assise, intellectuelle et matérielle, de son fonctionnement et ne pourrait s'en dégager qu'au risque d'y laisser sa substance. L'argument métaéthique de Miller sur l'origine «contextuelle ${ }^{11}$ » des principes dessine ici les frontières de la justice et inspire l'argument éthique en faveur de la préférence nationale. Autrement dit, la justice sociale ne peut s'émanciper des conditions dans lesquelles elle est apparue car elle reste liée à elles pour des raisons nécessaires et non pas seulement historiquement contingentes. C'est pour cela que Miller ne peut accepter de faire de la justice mondiale une question de justice sociale à une simple différence d'échelle près ${ }^{12}$. Il nous faut identifier le statut de l'argument historique mobilisé ici, examiner sa vulnérabilité potentielle à des récits concurrents sur les conditions qui ont rendu et continuent de rendre possible la justice sociale. Cela requiert aussi de préciser ce qui, dans l'idée de nation, a pu se révéler aussi indispensable pour son établissement.

\section{La naissance d'un concept}

Miller est l'un des rares auteurs, dans la littérature abondante qui a suivi la publication de la Théorie de la justice de Rawls en $1971^{13}$, à insister autant sur l'émergence historique du concept de justice sociale. Il montre notamment que ses premiers théoriciens à la fin du XIX ${ }^{\mathrm{e}}$ siècle, comme Leonard Trelawny Hobhouse ou Westel Woodbury Willoughby, ont révélé la dimension organique de la société pour mettre en avant que l'épanouissement de chacun exigeait la coopération de tous les autres. Il fallait en effet dépasser l'illusion individualiste contenue dans les représentations du corps social pour pouvoir exiger que des institutions régulent, compensent, réparent les destins inévitablement interdépendants de ses membres. Rendre visible le réseau institutionnel qui sous-tendait le rapport entre les contributions et les conditions de vie des individus était la première étape pour imaginer pouvoir confier à des institutions le soin de répartir les bénéfices et les charges de la coopération sociale ${ }^{14}$. Miller ne fait au fond ici que reprendre l'idée d'une corrélation entre la naissance d'une compréhension sociologique des interactions humaines et celle d'une

\footnotetext{
11. Sur son « contextualisme », voir D. Miller, Justice for earthlings, Cambridge/New York, Cambridge University Press, 2013, chapitre 2, où il l'oppose à l'universalisme, au conventionnalisme et au relativisme.
}

12. Ibid., p. 166.

13. J. Rawls et C. Audard, Théorie de la justice, Paris, Seuil, 1997.

14. Miller, op. cit., 1999, p. 4. 
exigence positive de solidarité instituée entre les personnes ${ }^{15}$. Cette exigence supposait également la naissance de l'économie politique comme science, c'est-à-dire l'acquisition d'outils heuristiques pour comprendre que la répartition des biens sociaux n'était pas le fruit d'un processus aléatoire mais répondait à des lois et s'organisait selon des institutions identifiables (la propriété, le travail, le capital).

L'idée de nation sert, dans la pensée de Miller, à incarner cette dimension collective dans laquelle s'enracinent les existences pour saper l'illusion du self-made-man qui serait le seul propriétaire légitime des fruits de son travail ${ }^{16}$. Le cadre national révèle la solidarité des activités individuelles et soutient la revendication légitime de répartir les bénéfices et les charges issus de cette coopération. Le sentiment commun d'appartenance encouragé par la nationalité sous-tend enfin l'obligation de s'occuper du bien-être (welfare) de ses concitoyens ${ }^{17}$. Miller montre pour cela, dans un livre de 1989, la nécessité de réhabiliter le sens moral de la communauté nationale. Avant d'inscrire ses travaux sur la justice distributive dans la lignée de la Théorie de la justice de Rawls, il défendait dans cet ouvrage le modèle d'un « socialisme de marché ${ }^{18}$ », à mi-chemin entre le capitalisme et le socialisme, qui combine une économie de marché et un contrôle social sur la distribution des richesses et les conditions de travail. De tels principes, ajoutait-il, ne peuvent toutefois prendre place qu'à l'intérieur d'une communauté cohérente. Il faut en effet qu'existe une représentation solidaire $\mathrm{du}$ corps social pour que la thèse d'une production collective de la valeur devienne convaincante.

Il suggère donc que le sentiment national contribue au succès des théories favorables à la redistribution des biens car il rend visible les processus nécessairement collectifs de leur création. Avoir conscience de n'être qu'un membre - appartenant au même titre que les autres à une société nationale - facilite la compréhension de l'inextricable solidarité entre les actions individuelles et crée les conditions nécessaires à la mise en place d'un mécanisme positif, institutionnel, de solidarité. La critique de la conception individualiste de la production et donc de la distribution des biens se fait, de l'aveu de Miller, sur la base d'une conception que l'on pourrait dire «communautarienne ${ }^{19}$ » de la justice : les principes doivent s'enraciner dans une communauté soudée par un sentiment d'appartenance et des représentations communes. Elle se distingue toutefois par son contenu spécifiquement national.

15. Idée mise en avant notamment par Pierre Rosanvallon dans P. Rosanvallon, La société des égaux, Paris, Seuil, 2011, p. 260 et suiv.

16. D. Miller, Market, State and Community, Oxford, Clarendon press, 1989, p. 229.

17. Ibid., p. 246.

18. Miller, op. cit., 1989.

19. Il ne voit pas d'objections à qualifier sa thèse de « communautarienne » (ibid., p. 251). 


\section{Les conditions d'émergence de la justice sociale}

Le rôle propice que joue le sentiment d'appartenance nationale dans les consciences collectives ne relève toutefois pas seulement d'une idée sur les conditions intellectuelles préalables à l'émergence d'un concept. Il appartient à une thèse plus générale sur les facteurs qui ont permis l'émergence de la justice sociale. Cette interrogation historique recèle un enjeu philosophique fort chez Miller puisqu'elle vise à arbitrer entre des compréhensions concurrentes du domaine adéquat dans lequel penser la justice distributive. L'un des arguments pour contrer les ambitions cosmopolitiques des théories de la justice est en effet de démontrer que les conditions qui rendent possibles ou qui justifient la justice sociale à l'échelle des États ne sont pas remplies à l'échelle mondiale. Puisque les cosmopolitiques font notamment valoir des arguments historiques comme l'ouverture inédite des frontières ou l'intensité croissante du commerce entre les peuples ${ }^{20}$ pour défendre leurs conceptions, on peut leur opposer que ces éléments ne suffisent pas à créer les « circonstances de justice » qui ont rendu et rendent encore possible l'institution d'une justice sociale.

Pour que l'argument historique se révèle efficace dans cette démonstration, il faut à la fois identifier les facteurs propices au développement de la justice sociale et montrer en quoi ils ne peuvent se retrouver que dans un contexte national. L'idée défendue par Miller est que l'on ne peut se contenter d' «étendre» des principes qui valent à l'intérieur d'un pays à des relations transnationales ou internationales ${ }^{21}$ sans tenir compte de leurs conditions d'apparition habituelles. Il ne se satisfait toutefois pas de donner un argument réaliste, ne tire pas la validité de sa thèse de la valeur intrinsèque que revêtirait l'organisation du monde en États telle qu'elle existe. Il envisage au contraire de réviser les frontières de la justice, mais prétend que l'examen des facteurs pertinents pour le faire renforce sa conception nationale.

Le problème est donc de déterminer «quels sont les traits de l'État-nation qui importent lorsqu'on s'occupe de justice ${ }^{22} »$. L'histoire a facilité la tâche des défenseurs de la thèse nationale, ajoute-t-il, puisque la justice sociale est née dans ce contexte et y demeure confinée. Mais il reste à identifier les caractéristiques nationales qui ont réellement joué un rôle dans cet établissement et, si elles ne peuvent effectivement pas s'exporter hors de la nation, qui justifient de maintenir la justice sociale dans ces limites. Miller énumère trois caractéristiques de la nation qui sont pertinentes pour expliquer son émergence.

20. La première formulation de cet argument sur l'existence de conditions historiques qui appellent une justice distributive mondiale se trouve dans C. Beitz, Political theory and International Relations, Princeton, N. J., Princeton University Press, 1979.

21. D. Miller, op. cit., 2013, p. 166.

22. Ibid., p. 170. 
Premièrement, il existe à l'intérieur d'une nation un consensus sur la «métrique $e^{23}$ » de la justice. Il faut en effet que les membres d'une société s'entendent sur ce que signifient les biens sociaux à partager et sur les justes principes de leur répartition. Deuxièmement, les membres de la communauté se voient mutuellement comme formant un groupe de référence : ils se comparent spontanément entre eux, se plaignent de leur salaire, jugent l'intensité de leur travail par rapport à leurs concitoyens et non par rapport à des étrangers. Cela relève d'un «fait psychologique ${ }^{24}$ » selon Miller. Autrement dit, la nation est le lieu des sentiments de rivalité comme de solidarité et, dans un esprit rousseauiste, Miller défend qu'ils fonctionnent de concert. Enfin, troisièmement, l'activité économique et les institutions sociales doivent être soumises à une régulation coercitive de la part d'un État pour que la distribution des bénéfices et des coûts se conforme aux principes de justice reconnus collectivement.

Chacune de ces caractéristiques prises indépendamment ne suffit pas à constituer des circonstances de justice. Mais la nation les réunit toutes trois, ce qui explique que ses frontières aient dessiné les contours de la justice sociale. Ici et maintenant, écrit Miller en 2013, on doit donc continuer à la penser à l'intérieur des communautés politiques nationales. Le lien national semble fonctionner dans sa thèse comme la cause historique de la justice sociale. Mais qu'entend-il exactement par nationalité ? Il faut sans doute remonter à des textes antérieurs pour trouver une réponse à cette question.

\section{Quel rôle attribue-t-on exactement au concept de « nationalité » lorsqu'on en fait le ciment originel et nécessaire de la justice sociale ?}

\section{Elle n'est ni une « citoyenneté sociale »...}

La thèse la plus évidente pour expliquer cette coïncidence entre les frontières de l'État-nation et la justice sociale serait de montrer que les droits sociaux découlent du statut de citoyen d'un État. Si la nationalité est la base qui donne droit à des bénéfices sociaux, on comprend que ceux-ci soient strictement associés aux citoyens d'un pays et donc confinés à l'intérieur de ses frontières. Cette conception statutaire des droits sociaux est défendue par Thomas Humphrey Marshall dans Citizenship and Social Class ${ }^{25}$. Il existe, selon lui, trois évolutions décisives du statut de citoyen : l'acquisition des droits civils, puis des droits politiques (le droit d'élire et de se faire élire) et enfin des droits sociaux. Dans ce contexte, la garantie de conditions de vie décentes aux citoyens se comprend comme la réalisation de ce qu'impliquent

23. D. Miller, op. cit., 2007, p. 56.

24. D. Miller, op. cit., 1999, p. 18.

25. T. H. Marshall, Citizenship and Social Class, and Other Essays, Cambridge, Cambridge Universtiy Press, 1950. 
les droits politiques: nul ne saurait être autonome politiquement sans l'être économiquement ${ }^{26}$. Si chacun de ces acquis fonctionne comme l'élargissement du statut du citoyen, il est parfaitement logique que la nationalité reste la qualité qui définit la base sur laquelle s'établissent ces droits et l'ensemble, national, de leur application.

Miller refuse toutefois cette hypothèse fondée sur l'enrichissement progressif de droits individuels. En discutant la théorie de Marshall, il réaffirme que ce qu'il cherche à saisir, dans les conditions de possibilité de la justice sociale, c'est précisément ce qui excède la définition légale des droits et qui relève de l'implication des citoyens, de leurs croyances, de leurs conduites, de leur sentiment d'appartenance et de leur intérêt pour le bien commun $^{27}$, bref, de tout ce qui est « national » et non seulement politique ou juridique dans l'appartenance de l'individu au groupe ${ }^{28}$. Certes les acquis sociaux sont maintenant inclus dans la définition de la citoyenneté mais l'État providence était d'abord l'expression d'une solidarité nationale ${ }^{29}$ et non seulement le produit d'une identité citoyenne.

Il s'exposerait en effet à la menace cosmopolitique s'il retraçait la généalogie de la justice sociale à partir de l'acquisition de droits individuels. Inscrits dans la tradition des droits du citoyen, les «droits sociaux » sont compris comme l'enrichissement de bénéfices qui sont dus aux hommes en vertu de leur citoyenneté mais indépendamment de leur inscription dans un imaginaire national particulier. Si l'on suit Catherine Colliot-Thélène dans La démocratie sans démos, l'interprétation "nationale » de la citoyenneté est postérieure aux textes révolutionnaires dans lesquels «les droits des citoyens n'étaient pas réservés aux nationaux ${ }^{30} »$. Autrement dit, le territoire national pourrait n'être que la structure dans laquelle le peuple politique n'a eu d'autre choix que de se « couler $^{31} »$ et non une caractéristique consubstantielle à la définition de la citoyenneté politique et sociale. Cela permet de délier conceptuellement les acquis statutaires de la citoyenneté de son caractère strictement national ${ }^{32}$. Voilà précisément la thèse que Miller

26. Miller reconnaît que certains biens sont nécessaires à l'exercice égal de la citoyenneté mais il considère que la réalisation de l'égalité politique réelle ne suffit pas à rendre compte de ce qui motive la justice sociale (D. Miller, op. cit., 1989, p. 246-247 - D. Miller, op. cit., 1999, p. 250).

27. D. Miller, op. cit., 1989, p. 247.

28. D. Miller, Citizenship and national identity, Cambridge, UK-Malden, MA, USA, Polity Press/Blackwell Publishers, 2000, p. 82-83.

29. Miller, op. cit., 1999, p. 31.

30. C. Colliot-Thélène, La démocratie sans démos, Paris, PUF, 2011, p. 97. La Déclaration de 1789 distingue bien les droits de l'homme de ceux du citoyen, mais ces derniers valent, en théorie, pour tous les hommes puisqu'ils ont tous le droit naturel d'être citoyen d'une nation libre.

31. Ibid., p. 96.

32. C'est ce que suggèrent également les défenseurs de droits civils, politiques et sociaux pour les migrants sur un territoire étranger. Voir Y. Soysal, Limits of citizenship, Migrants and postnational membership in Europe, Chicago et Londres, The University of Chicago Press, 1994. 
entend contrer dans son argument général contre l'extension mondiale de la justice.

\section{... ni une identité romantique.}

Miller ne donne pas pour autant une définition romantique de la nationalité comme caractère ou comme âme nationale. Elle relève certes d'une identité commune, mais ne suppose pas une homogénéité ethnique ou culturelle. La nationalité est incarnée, elle désigne l'expérience subjective d'une «culture publique ${ }^{33}$ » qui fonctionne comme une médiation entre les intérêts particuliers et l'engagement public : indépendamment de leurs convictions privées, les citoyens d'une nation partagent certaines croyances, notamment sur leurs origines et leur histoire. Peu importe que ces dernières soient imaginaires, comme le suggère Benedict Anderson ${ }^{34}$, elles traduisent un accord sur ce qu'est la communauté et sur la façon de formuler les principes qui doivent la gouverner.

C'est précisément cette convergence d'imaginaires qui rend possible l'existence d'un consensus sur la valeur des biens sociaux et sur la pertinence des différents critères de justice. Elle crée une entente sur ce que signifient la solidarité, l'effort ou le travail. Miller juge sa définition de la citoyenneté «républicaine ${ }^{35}$ » dans la mesure où elle suppose un engagement civique, une participation à la vie publique motivée par des valeurs communes. Elle ne pourrait se réduire à un amour froid pour des institutions politiques ou à un strict respect pour le pluralisme des valeurs. Malgré les différentes cultures privées qui sont admises à l'intérieur d'une même culture nationale ${ }^{36}$, celle-ci implique un sentiment d'appartenance fort qui sert de terreau aux principes de justice.

Ces définitions de la nationalité permettent de suggérer que dans l'argument de Miller, deux caractéristiques sont pertinentes pour montrer l'élaboration nécessairement nationale de la justice sociale. Premièrement, elle est un sentiment subjectif qui contient la conscience collective d'appartenir à un ensemble délimité par des frontières. Miller, à la suite de Michael Walzer, insiste en effet sur la notion de «limite» contenue dans la définition de la communauté comme condition préalable à toute représentation de la justice ${ }^{37}$. Deuxièmement, la nationalité désigne une communauté d'imaginaires qui rend possible un consensus sur la

\footnotetext{
33. D. Miller, op. cit., 1995 , p. 6 ; p. 25.
}

34. Miller cite à cet égard la thèse de Benedict Anderson dans B. Anderson, L'imaginaire national: réflexions sur l'origine et l'essor du nationalisme, Paris, La Découverte/Poche, 1996 (Miller, 1995, chapitre 2).

35. Miller, op. cit., 2000, p. 82.

36. Miller considère que son approche reste à ce titre « libérale » en raison de l'attention qu'il accorde à la distinction entre public et privé (D. Miller, «The Ethical Significance of Nationality », Ethics, 98, 1988, p. 657).

37. Miller, op. cit., 2013, chapitre 6. 
signification et la répartition des biens sociaux. Sans cette conscience d'être une partie dans un tout, les individus ne comprendraient pas que la coopération sociale crée une interdépendance que seule une organisation nationale peut réguler. Sans un accord sur le sens de la justice, il aurait été impossible de faire accepter collectivement des mécanismes de solidarité.

\section{L'imbrication des arguments éthiques et métaéthiques pour démontrer le rôle de la nationalité dans l'institution de la justice sociale}

Il faut toutefois isoler le rôle de la nationalité ainsi conçue dans l'argument général de Miller. Il pourrait sembler plus intuitif de se contenter de dire que, le monde étant ce qu'il est, les États constituent aujourd'hui les seules puissances souveraines. Quelle que soit la nature du ciment national, il existe des communautés politiques fermées dans lesquelles des institutions coercitives sont capables d'appliquer des principes de justice. Que leur application repose sur une légitimité démocratique, sur des sentiments de solidarité réels, sur un consensus culturel, cela peut sembler n'avoir pas tellement d'importance ${ }^{38}$.

Pour Miller, le facteur «national», entendu comme expérience subjective d'une identité commune, est pourtant tout aussi important que l'existence de mécanismes de coercition. Cela s'explique par la valeur morale qu'il accorde à la nationalité. Il lui reconnaît en effet une «signification éthique ${ }^{39}$ » et s'engage ainsi du côté du particularisme éthique: des devoirs particuliers émergent des relations spécifiques que nous entretenons avec nos proches (special duties ${ }^{40}$ ). Il s'inspire ici de la critique de Michael Sandel contre le modèle de l'agent rationnel rawlsien pour montrer l'importance de la proximité, du contexte relationnel dans la motivation morale. Cela ne veut pas dire pour autant que Miller envisage la nation comme une extension de la famille. Au contraire, le sentiment national est ce qui nous sort de la partialité de nos relations familiales, amicales ou communautaires et nous permet d'adoucir le conflit qui oppose nos préoccupations égoïstes et notre souci de la chose publique ${ }^{41}$. La communauté nationale est donc le lieu intermédiaire qui convient pour les devoirs de solidarité : elle nous lie aux autres pour des raisons publiques, mais justifie les limites de ces devoirs. Il n'y a donc pas d'objection de

38. L'argument de la souveraineté est défendu par des opposants aux conceptions cosmopolitiques de la justice, voir par exemple T. Nagel, « The Problem of Global Justice », Philosophy \& Public Affairs, 2005, 33(2), p. 113-147 ou M. Blake, « Distributive Justice, State Coercion, and Autonomy », 2001, Philosophy \& Public Affairs, 30(3), p. 257-296.

39. D. Miller, op. cit., 1988.

40. Ibid., p. 649.

41. D. Miller, op. cit., 1999, p. 18. 
principe à ce que les services de l'État providence bénéficient exclusivement à ceux qui se trouvent sur le même territoire national ${ }^{42}$.

Cette valeur morale suppose à son tour un argument métaéthique sur l'origine des principes. Depuis cet article de 1988 jusqu'à son livre le plus récent, en 2013, Miller reprend du "contextualisme » de Walzer l'idée que les principes de justice distributive ne peuvent être dérivés de principes abstraits car ils dépendent de la signification sociale que leur reconnaît une communauté particulière. Ils présupposent une histoire et un langage communs, dans lesquels puisse s'exprimer cette valeur ${ }^{43}$. "Satisfaire les besoins fondamentaux » ne prend pas le même sens à Athènes ou pour une communauté juive du Moyen Âge, pour reprendre l'exemple de Walzer, car la nature du besoin ne va pas de soi et que l'on ne peut décider de principes pour répartir des biens sociaux avant d'avoir admis ce qui leur donnait un tel statut, c'est-à-dire en révélant le caractère historique et anthropologique de ces significations sociales ${ }^{44}$. "L'idée de justice sociale ne prend sens que si l'on suppose qu'il existe un large consensus sur la valeur sociale d'un ensemble de biens, de services, d'opportunités, en dépit des désaccords dans les jugements privés » écrit Miller ${ }^{45}$. Et ce consensus n'est possible que dans le contexte national, où l'on entend la même chose par « besoin » mais aussi par «travail » ou par «mérite » ${ }^{46}$. Un livre récent de Jean-Claude Barbier présente ainsi les problèmes de traduction de l'expression «État providence » des deux côtés de la Manche et suggère qu'il sera difficile d'avancer dans la «longue marche vers l'Europe sociale» tant le mot lui-même ne s'entend pas de la même façon ${ }^{47}$.

L'existence de l'État-nation comme contexte d'émergence fait donc office pour Miller d'un « présupposé » qui n'a pas seulement valeur de vérité historique. Selon sa thèse métaéthique, comprendre la valeur des principes de justice sociale, c'est en faire la généalogie. Et c'est dans la «nationalité » qu'ils s'originent et tirent leur sens. Cet engagement métaéthique n'est pas conservateur: il ne voue pas un culte à l'histoire ni ne défend un immobilisme politique. En revanche, il défend qu'on ne peut extraire des principes de leur contexte de justification et ajoute que celui de la justice sociale est historiquement national. Si l'on s'en tient aux deux caractéristiques énoncées, elles reposent sur un rapport de causalité et non seulement de coïncidence entre l'existence d'un sentiment national, défini comme conscience d'appartenir à un tout délimité et comme accord sur la signification des biens sociaux, et l'établissement d'une justice sociale.

42. D. Miller, op. cit., 1995, p. 11.

43. D. Miller, op. cit., 2013, p. 143.

44. M. Walzer, Sphères de justice, Paris, Seuil, 1983, p. 119.

45. D. Miller, op. cit., 1999, p. 8.

46. Ibid., p. 8.

47. J. -C. Barbier, La longue marche vers l'Europe sociale, Paris, Presses universitaires de France, 2008. 
On pourrait certes transposer des principes de justice sociale à l'échelle mondiale si ce qui importait dans son contexte d'émergence était l'existence de relations économiques répétées ou de contacts culturels forts. Mais c'est parce que le sentiment national a joué un rôle de choix dans cette construction que les ambitions cosmopolitiques sont vaines d'après Miller. La force de cette thèse, intuitive dans ses arguments, sensible au contexte, en est aussi sa faiblesse. En effet, il n'est pas sûr qu'elle survive à des récits alternatifs sur l'apparition de la justice sociale. Si c'est de façon plus contingente que la justice sociale est née dans le cadre de l'État-nation, leur lien devient plus lâche et le domaine d'application discutable. Pour le dire autrement, l'État-nation a certes été le contexte d'apparition de préoccupations sociales et le lieu où s'est développé le droit social. Mais Miller franchit un pas conceptuel important lorsqu'il passe de la nation comme arrière-plan historique, institutionnel, à l'idée de nationalité comme sentiment d'une communauté morale solidaire que seraient venu exprimer, traduire des principes positifs de solidarité.

La longue marche vers l'institution des droits sociaux, la redistribution des richesses, l'accès à l'éducation n'a sans doute pas été si clairement médiatisée par l'idée de nation comme représentation d'une communauté de sens délimitée et solidaire. Et si cette coïncidence historique entre justice sociale et État-nation à la fin du XIX ${ }^{\mathrm{e}}$ siècle et au milieu du $\mathrm{XX}^{\mathrm{e}}$ siècle ne traduisait pas un consensus national sur la justice ni un sentiment de solidarité mais des rapports de force, des représentations contradictoires du corps social, est-ce que cela ne remettrait pas en cause la valeur morale des principes de justice ainsi que le dessin de ses contours historiques?

\section{Les récits alternatifs sur les conditions d'émergence de la justice sociale}

Pour discuter cette thèse, il faudrait présenter les éléments d'un débat historiographique sur le rôle qu'a pu jouer l'idée de nation, ou plus précisément le sentiment national, dans cette apparition. Dans le cadre de cette étude philosophique, on peut au moins suggérer que la confrontation de la thèse de Miller à quelques hypothèses suffit à relativiser un présupposé qu'il conçoit comme évident ${ }^{48}$, à dédramatiser l'importance de cette identité nationale pour la justice sociale et donc, sans doute, à réviser les frontières de son application légitime.

Précisons d'abord que Miller suit l'opinion de nombreux auteurs qui ont mis en avant la coïncidence entre des circonstances propices aux sentiments patriotiques et la mise en place du système de protection sociale.

48. Les premières théories de la justice distributive «présupposaient simplement qu'ils parlaient d'une justice à l'intérieur des frontières de communautés organisées politiquement, en d'autres termes, ce que nous appelons aujourd'hui États-nations » (Miller, 1999, p. 4). 
Pierre Rosanvallon a par exemple mis en lumière l'influence de l'expérience des deux guerres mondiales sur le développement des États providence européens. Il s'appuie sur les écrits de William Beveridge, fondateur de l'État providence anglais, pour montrer le lien fondamental entre les manifestations de l'engagement patriotique dans la guerre et la reformulation du contrat social conçu comme «dette » de l'État à l'égard de ses anciens soldats $^{49}$. Le champ de bataille aurait rendu visible l'égalité de conditions entre les hommes, et donc acceptable l'idée d'une socialisation des risques et des avantages communs en vue de leur offrir à tous une existence décente. Rosanvallon s'interroge alors sur la nécessité d'un « équivalent moral » au patriotisme issu de cette histoire pour refonder l'État providence. Il déplore en effet que la disparition de l'esprit civique ait privé la solidarité de son assise: «Ce n'est pas seulement la mobilisation qui manque, c'est le substrat de celle-ci, c'est-à-dire la nation $»^{50}$.

L'hypothèse d'une solidarité gonflée par les sentiments nationaux exacerbés aux cours des guerres mondiales bénéficie d'arguments historiques probants. La Constitution de 1946 donne certainement la première formulation aussi aboutie des droits économiques et sociaux en France. «La nation proclame la solidarité et l'égalité de tous les Français devant les charges qui résultent des calamités nationales » dit le Préambule de 1946, établissant un lien substantiel entre égalité et solidarité et s'inspirant directement des programmes du Conseil national de la Résistance $^{51}$. Les théories convergent généralement pour faire remonter l'émergence des États providence européens dans leur forme actuelle aux lendemains de la Seconde Guerre et soulignent ainsi le facteur national - à la fois comme sentiment patriotique, comme consensus idéologique et comme intervention étatique - dans leur établissement.

Il existe cependant des récits alternatifs sur le rôle du sentiment national dans la construction des politiques sociales. Nous allons nous contenter ici de confronter cette thèse à deux autres versions : le développement d'une mutualisation des risques par les travailleurs et le rôle des luttes sociales dans l'acquisition de droits sociaux.

\section{L'assurance comme mutualisation des risques}

L'histoire de la protection sociale, en France, est par exemple ancrée dans les corporations de l'Ancien Régime puis dans des sociétés de secours

49. P. Rosanvallon, La nouvelle question sociale, repenser l'État-providence, Paris, Seuil, 1995 , p. 50.

50. Ibid., p. 73.

51. Voir C. Bec, La sécurité sociale : une institution de la démocratie, Paris, Gallimard, 2014. Voir aussi Dominique Schnapper qui insiste sur l'influence des programmes de la Résistance sur l'État providence français et sur celle de l'économie de guerre sur le système de santé britannique (D. Schnapper, La démocratie providentielle, Paris, Gallimard, 2002, p. 41-42). 
mutuels organisées par profession. Une charte autorise la mutualité en 1898 mais la laisse entre les mains de ces sociétés qui assuraient les cotisants contre les risques encourus par la maladie, la vieillesse, l'invalidité, voire qui organisaient leurs funérailles. Nul ne songeait à étatiser la mutualité et Colette Bec montre que les sénateurs français étaient même réticents à l'idée de rendre obligatoire la souscription aux assurances au travail ${ }^{52}$. Ce n'est qu'après la Première Guerre mondiale que l'État est désigné comme l'agent capable de prendre en charge ces missions sociales et que leurs bénéficiaires deviennent universels. Le motif à l'origine de ces pratiques était donc moins pour leurs membres d'exprimer un sentiment de solidarité national que de coordonner leurs intérêts rationnels pour se prémunir des risques créés par le travail en les mutualisant, souvent à l'intérieur d'une catégorie socioprofessionnelle et selon un mécanisme contractuel et assurantiel. Le principe de socialisation des risques était plutôt une glorification de la responsabilité des individus à prévoir et à anticiper leur avenir qu'une manifestation de la solidarité nationale.

Ces assurances ne constituent certes qu'un embryon de protection sociale. Mais sur le plan des motivations, cher à Miller, remarquons qu'elles s'enracinent clairement dans une échelle plus restreinte que le territoire national et relèvent au mieux d'une solidarité locale. Cette idée est encore plus probante pour le système d'assurances sociales bismarckien, organisé par Länder, dans lequel les indemnités étaient clairement les contreparties de cotisations versées par les travailleurs et qui pourrait difficilement s'interpréter comme la traduction institutionnelle d'un sentiment d'appartenance à une nation en pleine construction. Le système beveridgien, dans lequel les cotisations financent un fonds qui n'est pas réservé à ceux qui ont cotisé mais fournit à tout membre de la société un niveau minimum de ressources, requiert sans doute davantage la médiation d'une conception délimitée et solidaire de la communauté nationale ${ }^{53}$.

On peut plus généralement opposer à cette première objection historique que ce n'est justement qu'à la condition du passage d'une logique assurantielle à une logique solidaire, de transferts dépendants des cotisations versées à des transferts qui en sont indépendants, bref de l'établissement d'un projet politique, que l'on peut réellement parler de justice sociale ${ }^{54}$. Toutes les formes antérieures de solidarité ne mériteraient pas d'être qualifiées de la sorte. La nationalisation du système de protection sociale, consubstantiel à son caractère obligatoire, en marquerait ainsi l'acte de

52. Voir C. Bec, La sécurité sociale : une institution de la démocratie, op. cit., p. 56.

53. Pour une discussion des différentes justifications à l'œuvre dans ces modèles, voir P. Van Parijs, «Au-delà de la solidarité. Les fondements éthiques de l'État-providence et de son dépassement», in Repenser la solidarité. L'apport des sciences sociales, Serge Paugam (dir.), Paris, PUF, 2006, p. 124-146.

54. Voir sur ce point le commentaire du livre de Colette Bec par Jean-Fabien Spitz, « Solidarité ou assurance » sur le site La vie des idées. 
naissance ${ }^{55}$ et l'idée de nation comme ensemble solidaire dont l'État est le garant légitime serait alors le ressort le plus important pour rendre compte de ses fondements. Cette idée est précisément celle que défend Miller pour montrer que le ciment requis pour la justice sociale ne peut se résumer à l'agrégation de calculs rationnels en vue d'un «avantage mutuel ${ }^{56}$ ». Il en conclut que seule la nationalité donne l'assise morale requise pour la logique solidaire, là où d'autres la placent dans une conception démocratique de la citoyenneté sociale telle que Miller l'a rejetée (voir supra, p. 6-7). On peut toutefois garder de cet exemple historique l'idée que les systèmes de protection voient le jour dans des structures qui ont davantage trait à l'affiliation professionnelle qu'à l'appartenance nationale.

\section{Le rôle des luttes sociales dans l'acquisition de droits sociaux}

Quant à la deuxième caractéristique pertinente chez Miller, c'est-à-dire l'accord sur les biens et les critères de justice, il semble qu'elle soit mise à l'épreuve par l'hypothèse selon laquelle la nationalisation des systèmes de protection sociale - par ailleurs contrastée selon les pays occidentaux ${ }^{57}$ résulte davantage de conflits sociaux que d'un consensus culturel ancré dans l'imaginaire national.

L'acquisition des droits sociaux a en effet autant résulté de compromis, voire de conflits politiques, que d'un consensus national sur la signification des biens. Les luttes sociales ont pu précisément avoir pour objet de s'opposer au prétendu consensus national sur le sens de la justice. Cette thèse vaut d'ailleurs pour les deux périodes clé de la mise en place des mécanismes de solidarité : la généralisation des systèmes d'assurances sociales à la fin du XIX ${ }^{\mathrm{e}}$ siècle et la naissance des États providence européens après la Deuxième Guerre mondiale. Il suffit d'observer la corrélation entre les périodes propices au progrès social et celles où pesait fortement la menace contestataire. Le modèle bismarckien traduit par exemple le nécessaire compromis des classes dominantes avec les classes laborieuses en Allemagne à la fin du $\mathrm{XIX}^{\mathrm{e}}$ siècle et les écrits de Bismarck attestent que l'objectif de ses réformes était de gagner les classes populaires à la cause conservatrice et de prévenir le désordre public ${ }^{58}$. L'institution de l'État providence en France en 1945 peut, dans la même veine, s'interpréter

55. « On passe d'une protection conçue en termes individuels ou catégoriels à une véritable politique de Sécurité sociale engagée par les pouvoirs publics, l'obligation exprimant clairement la légitimité du pouvoir étatique ». C. Bec, La sécurité sociale : une institution de la démocratie, op. cit., p. 118.

56. D. Miller, op. cit., 1995, p. 72.

57. Voir la typologie de G. Esping-Andersen, Les trois mondes de l'État providence, Paris, PUF, 2007, p. 41-42.

58. C. Colliot-Thélène, La démocratie sans démos, op. cit., p. 176. 
comme le compromis que le capitalisme a dû faire pour répondre à la menace que représentait le communisme, comme idéologie et comme parti politique ${ }^{59}$.

De nombreux auteurs ont insisté sur les conflits entre classes sociales à l'origine des progrès sociaux et l'importance de l'une d'entre elles dans le triomphe d'un système de solidarité. C'est généralement la classe ouvrière qui tient le rôle principal dans ce récit, pour avoir su imposer de meilleures conditions de travail, ainsi que des mécanismes de redistribution et de protection sociale. La corrélation entre l'apogée de la démocratie sociale et l'ère industrielle pourrait témoigner de l'influence du poids politique et économique de la classe ouvrière à la fin du $\mathrm{XIX}^{\mathrm{e}}$ et au début du $\mathrm{XX}^{\mathrm{e}}$ siècle sur l'obtention d'acquis sociaux. Il fallait qu'elle soit essentielle à l'économie nationale et détentrice d'un pouvoir politique pour que ses luttes trouvent un écho auprès des élites ${ }^{60}$. Des travaux ont certes relativisé le poids de la classe ouvrière dans la mise en place des États providence, en insistant davantage sur ses accords passés avec d'autres classes sociales, comme dans le cas de la coalition "rouge verte» avec la paysannerie à l'origine du modèle suédois par exemple ${ }^{61}$ ou en montrant le rôle décisif des classes moyennes $^{62}$. Tenons-nous en à l'idée que toutes ces hypothèses sont sensibles aux conflits entre classes ou entre catégories sociales qui apparaissent à la source du succès de politiques plus égalitaires et qu'elles mettent principalement l'accent sur les clivages et non sur les consensus nationaux.

Si l'on cherche à donner un sens éthique, parce qu'historique, à la nation, il faut donc s'entendre sur ce qu'on lui reconnaît comme rôle dans l'histoire. Que les systèmes de Bismarck ou de Beveridge soient empreints des traditions et de l'histoire particulières à chaque nation, c'est incontestable. En revanche, si l'on conçoit la nationalité comme une cause historique à l'origine de la justice sociale pour montrer l'impossibilité de l'étendre hors des frontières, ces hypothèses se révèlent plus fragiles. La conception de la nationalité chez Miller admet certes des évolutions et les différentes luttes sociales peuvent être lues comme autant d'éléments constitutifs de ce qu'il nomme la « culture publique ${ }^{63} »$. Il ne donne en effet pas une définition figée ou parfaitement consensuelle de l'assise nationale nécessaire à la mise en place de principes de justice. On peut toutefois se demander ce qu'il reste de spécifiquement national dans les conditions de possibilité de la démocratie sociale. Selon les deux caractéristiques envisagées, il ne s'agit ni de la conscience d'appartenir à une communauté

59. D. Schnapper, La démocratie providentielle, Paris, Gallimard, 2002, p. 45-46.

60. C. Crouch, "The Parabola of Working Class Politics», The Political Quarterly, Volume 70, Issue Supplement s1, p. 69-83, Special Issue, 1999.

61. G. Esping-Andersen, Les trois mondes de l'État providence, op. cit., p. 30-33.

62. Voir P. Balwin, The Politics of Social Solidarity: Class Bases of the European Welfare State, 1875-1975, Cambridge University Press, 1990.

63. D. Miller, op. cit., 1988 et D. Miller, op. cit., 1995. 
délimitée, puisque les systèmes d'assurance et de solidarité n'ont pas attendu la nationalisation de la protection sociale pour apparaître, ni de l'existence d'un univers de signification sociale, étant donné les conflits qui ont été nécessaires à l'intérieur des États-nations pour faire accepter le sens des biens sociaux et des principes de répartition exigeants. Que les acquis sociaux fassent aujourd'hui office de patrimoine national, comme Miller semble le suggérer pour le système de santé canadien ${ }^{64}$, cela relève de la proposition inverse et cela n'implique pas les mêmes conclusions pour les ambitions supranationales des auteurs cosmopolitiques.

\section{Conclusion}

L'enjeu de l'argument historique reste en effet pour Miller de consolider une thèse normative sur l'applicabilité des principes de justice hors des frontières nationales. Que dire alors, si l'on renverse le raisonnement, de l'existence à l'échelle supranationale de certains phénomènes que ces hypothèses alternatives comptent parmi les facteurs déterminants pour expliquer les progrès historiques en matière de justice sociale? Il n'y a certes pas d'autres pouvoirs souverains que les États-nations, le monde étant ce qu'il est, et aucune institution capable d'engager l'équivalent d'une nationalisation de la solidarité à l'échelle mondiale. On ne peut sans doute même pas aller, avec Charles Beitz, jusqu'à prétendre qu'il existe une société civile universelle ou jusqu'à déceler, en suivant Jürgen Habermas, l'apparition d'une opinion publique mondiale ${ }^{65}$. En revanche, il convient de souligner l'existence de revendications portées par des mouvements sociaux supranationaux, dont les préoccupations convergent vraisemblablement. Il faut attirer l'attention sur l'émergence d'un syndicalisme transnational comme c'est le cas dans l'industrie du textile (Clean Clothes Campaign est par exemple un syndicat actif dans une dizaine de pays européens) ou chez les ouvriers du transport (la Fédération internationale des transports est ainsi à l'origine du mouvement collectif en jeu dans l'affaire Viking en Europe ${ }^{66}$ ). À partir des campagnes contre les sweatshop, c'est-à-dire les protestations aux États-Unis contre les conditions de travail des producteurs à l'étranger de marchandises consommées sur leur territoire, Iris Marion Young s'interroge sur les différentes échelles pertinentes de justice et affirme qu'elles sont mondiales quand les connexions sociales à l'origine des injustices sont elles-mêmes mondiales ${ }^{67}$. Si ces manifestations de solidarité

64. D. Miller, op. cit., 1995, p. 95.

65. J. Habermas, L'intégration républicaine, Paris, Fayard, 1998, p. 168.

66. Case C-438/05 International Transport Workers' Federation and Finnish Seamen's Union v Viking Line ABP and OÜ Viking Line Eesti [2007] 2007 ECR I-10779.

67. Voir I. M. Young, «Responsibility and Global Labor Justice», Journal of Political Philosophy, 12(4), (2004), p. 365-388 et J. Habermas, op. cit. 
ou ces revendications comptent bien parmi les facteurs qui ont rendu possible le triomphe des démocraties sociales européennes à l'échelle nationale, et que la nationalité comme culture publique est toujours révisable, à partir de quand pourra-t-on dire que ces éléments comptent autant que l'idée nationale dans les conditions qui définissent des « circonstances de justice»? Réciproquement, la crise des États providence en Europe pourrait remettre en cause le présupposé national de la justice sociale et suggérer des pistes pour lui substituer des fondements sans doute moins enracinés historiquement, mais plus solides aujourd'hui.

\section{Références bibliographiques}

Miller, D., « The Ethical Significance of Nationality », Ethics, 98, 1988.

Miller, D., Market, State and Community, Oxford, Clarendon press, 1989.

Miller, D., On nationality, Oxford/New York, Clarendon Press/Oxford University Press, 1995.

Miller, D., Principles of social justice, Cambridge, Mass., Harvard University Press, 1999.

Miller, D., Citizenship and national identity, Cambridge, UK-Malden, MA, USA, Polity Press/Blackwell Publishers, 2000.

Miller, D., National Responsibility and Global Justice, Oxford/New York, Oxford University Press, 2007.

Miller, D., Justice for earthlings, Cambridge/New York, Cambridge University Press, 2013. 